\title{
Main Trends in mHealth Use Scenarios
}

\author{
Sergey Balandin, Ekaterina Balandina, Yevgeni Koucheryavy, Vadym Kramar, and Oleg Medvedev
}

\begin{abstract}
The mobile-devices assisted healthcare and medical applications are expected to create the next breakthrough in demand for smartphones and mobile services. In the last decades we witnesses aging of populations and fast growth of healthcare related expenses. This leads to growth of demand for inexpensive and efficient health monitoring solutions. Mobile phone is the best positioned candidate device for managing aggregation and preliminary analysis of personal data, including information about health and physical activities. Because of that the idea of personal assistant device for health monitoring and early diagnostics of diseases is getting more and more popular. This paper discusses the main currently developed use cases and top trends in development of mobile healthcare use scenarios. In our studies we reviewed and analyzed broad variety of mHealth use cases, i.e., starting from monitors of the most relevant health parameters and updates personal medical record (e.g., blood pressure, ECG, breath activity, etc.), to advanced cloud systems that performs personalized diagnostics and recommendations on healthy behavior and individual medical smart systems that perform advance diagnostic of user's health and modeling of potential trends and negative developments.
\end{abstract}

Index Terms-mobile healthcare, mHealth use cases, health information management, human computer interaction, medical information systems, smart systems.

\section{INTRODUCTION}

In the last decades the mobile phones got wide circulation and become the most commonly used personal mobile devices. Thanks to advance of their functionality and convenience of use, the mobile device has already entered to many areas of human life and a person of the 21st century cannot anymore imagine comfortable life without a mobile phone. The most advance mobile devices combine a lot of different functions, which in the past were delivered by a number of specialized devices. In most cases each function

Manuscript received December 14, 2013; accepted December 15, 2013. Date of online publication: December 15, 2013.

Authors are grateful for DIGILE IoT SHOK program that provided required support of this research.

S. Balandin is with FRUCT Oy, Helsinki, Finland (e-mail: sergey.balandin@fruct.org).

E. Balandina is with Tampere University of Technology, Tampere, Finland (e-mail: ekaterina.balandina@tut.fi).

Y. Koucheryavy is with Tampere University of Technology, Tampere, Finland (e-mail: yk@tut.fi).

V. Kramar is with Oulu University of Applied Sciences, School of Engineering, Oulu, Finland (e-mail: vadym.kramar@oamk.fi).

O. Medvedev is with Moscow State University, Moscow, Russia (email: oleg.omedvedev@gmail.com). or group of functions is targeted to attract a certain group of users. For example, teenagers are attracted by entertainment functions, such as mp3-player, photo and video camera, plus access to social networks and so on. Another group of users is attracted by the high-speed network connection and possibility to access Internet from anyplace at anytime. We can continue this list further, as in most cases a new group of users is attracted when the mobile device addresses a new area of human needs and enhances additional functionality from some specialized device or group of devices.

Participants of the World Economic Forum 2013 have listed 20 drivers that influence current healthcare trends [1]. The most important are political, such as influence over lifestyles, and origin of governance; social, such as attitudes towards solidarity, and health culture; and technological, such as organization of the health innovation system, and access to health information. Among other drivers are social, such as state of economy, and income equality; and environmental, such as climate change, and population sanitation. The following three scenarios were developed to envision the health systems of the future and mHealth solutions appear to be essential in all of those scenarios.

Health incorporated scenario redefines boundaries of the health industry: governments' cutback on public services, liberalisation of market, new commercial products and services, and as result - new sense of conditional solidarity.

The new social contract scenario stipulates governments' responsibility for efficient driving of health systems and regulating organizations and individuals to live healthy life.

Super-empowered individuals scenario implies that citizens use a large variety of competing products and services to manage their own health, and governments try to address the consequences of such competitive market.

But what is the next major opportunity on this market? "If you think about the driving forces that are going to shape health care for the next 20 to 30 years, three things stand out: major aging in the population, massive growth of chronic disease, not enough caregivers," says Steven DeMello, director of health care at the Center for Information Technology Research in the Interest of Society at the University of California, Berkeley. DeMello says that mobile diagnosis and surveillance could help blunt the impact of changing demographic trends by recognizing health crises early, by providing connections for remote care and by giving patients enough information to gain control of their disorder [2]. 
In June 2012 the FCC assembled a group of leading wireless healthcare experts to assess the opportunities and challenges related to adoption of wireless health technologies and offer recommendations for the government to move forward in promoting adoption of mobile technology [3]. The report suggested that mHealth could improve patient care and create cost savings in multiple ways. But to foster an environment which enables providers to better understand patients' health trends over time, granting patients easier and increased access to their health information, while increasing efficiencies across the continuum of care, leading to more accurate diagnosis and treatment [4] will take time and resources.

According to the latest studies and forecasts [5], the ten key mobile health trends for 2013-2017 are as follows:

1. Smartphone user penetration will be the main driver for the mHealth uptake

2. mHealth applications will be tailored specifically for smartphones or tablets

3. mHealth applications will be native rather than webbased applications

4. mHealth niche stores will become the home of the 2nd generation of mHealth apps

5. Missing regulations are the main market barrier during the commercialization phase

6. Buyers will continue to drive the market

7. Applications will enter traditional health distribution channels

8. mHealth market will grow mainly in countries with high Smartphone penetration and health expenditure

9. 2nd generation mHealth applications will focus on chronic diseases

10. mHealth business models will broaden.

This list of trends was formulated based on opinions of 324 leaders and mHealth app publishers that have been interviewed. In the extension to the report it has been stated that mHealth application market development is happening through the following three phases. The first initial phase is over by now. The second phase is a commercialization phase, which is characterized by a significant increase of solutions, and appearance of new business models. The third phase will be reached in five years, when mHealth solutions will be a mass market (estimate is $50 \%$ of billions of smartphone and tablet users will have downloaded mHealth applications).

The second phase of the mHealth application market is also confirmed by the global use of medicines outlook through 2017 report [6] developed by the IMS Institute for Healthcare Informatics. As for specialised mobile applications, 20007 out of 43689 applications available were not quite relevant to health. Out of relevant, 7400 are designed for healthcare professionals, and 16275 are patient-oriented.
Devid Levi, the PwC’s Global Healthcare Leader, gives a statement [7] that they do not really think of mobile health as a separate industry or a separate sector, but rather as the future of health care, a deep part of what is a revolution of health care around the world. Among the major trends that already happening in healthcare and affecting development of mobile technologies, the following are mentioned:

1. Ageing populations and chronic illness can benefit from mHealth that improves access and quality, and brings innovation and cost reduction.

2. The foundations of industrialisation of healthcare that emerges the "Care anywhere" concept, where a use of mHealth is essential.

3. Personalisation, for which mHealth can offer personal toolkits for predictive, participatory and preventative care.

Our further study was much influenced and inspired by the information about huge recent progress in mHealth field and the above listed ideas and statements. The paper shall be seen as an attempt to make an overview and first step analysis of the available studies on mHealth use cases.

The paper is organized as follows. Section II provides description of the general emerging use cases for mobile healthcare. Section III is specifically focused on discussion of the use cases for new groups of users and users in developing countries. In Section IV we introduce the advanced use cases for mobile healthcare, which are based on use of smart systems. These solutions are particularly targeting most active part of society in developed countries, which are also very active users of most advanced mobile technologies. Finally, Section V initiates general discussion on the current status of mHealth industry and concludes the paper with a summary of the main finding of the study.

\section{EMERGING USE CASES}

The roadmap for the Digital Patient [8] defines five areas of technological challenges: generation of data and information, biomedical information management, modelling, user interface, and translation adoption. Essentially the majority of biomedical data is acquired in a traditional way in accordance to well-defined processes of primary and secondary care. But several important trends may be observed. These trends are the following:

Primary care: takes over part of the diagnosis currently performed in the hospitals due to advances in technology, automation of data acquisition and miniaturisation of devices.

Secondary care: a growing complexity of diagnostic systems and even more complex data require availability of sophisticated systems that advance effective usage and data transfer.

Home care: an emerging shift of data acquisition that in addition to biomedical data would be able to obtain lifestyle and behavioural information. It brings a higher demand to 
validation of data quality and reliability, as well as privacy and security.

Mobile care: an increasing proportion of data will be captured by co-located, wearable, transcutaneous and implantable sensors through a variety of devices and mobile applications not only at fixed locations, but also on a wayTherefore a special attention has to be given to a data provisioning.

Speaking of biomedical information management - the most important trend is on extracting data from Electronic Health Record (EHR) systems that provide storage of personal health data for specific use in dedicated databases, but the same information is very useful for the personalised care based on flexible and highly-customisable services. With continuously growing volumes of data sets and collections, their diversity and linkage, we will see more solutions that will be able to handle Big Data.

The modelling paradigm shifts from simple models presenting isolated biomedical systems and organs towards robust patient-specific models that are built using the longitudinal data, both across the disease time-course and the life-span. It is expected that such models will significantly advance prevention and treatment of disease [8]. The advanced modelling will require utilisation of diverse datasets from a variety of sources, cognitive intelligence of supporting systems, and sophisticated simulation techniques that are able to eliminate a gap of missing data or such measures that are not possible to obtain. Visualisation systems that allow a seamless immersion into a model from the highest level of observation (an avatar in a virtual world) until the finestgraining levels (nano-biomedicine) are yet to come [9].

The demographical and economical changes such as ageing of populations and saving on healthcare and associated social welfare have a big impact to a usage of medical systems. The real shift of medical care from medical institutions to the home environment is needed [8]. Taking into consideration a patient-centric nature of emerging health-care services, appearance of consumers' user interfaces allowing a restricted access to medical data or results of its processing (e.g., visualised representations) is one of the very important trends. Broad spectrum of users, devices, functionalities and applications use scenarios bring here a number of challenges that may be addressed by the extensions of existing tools to support time-varying, dynamic data and support multi-scale interactive visualization for data defined at different time scales (data defined across different spatial scales), extensions that support novel human computer interaction and interactive visualization, effective information retrieval, and seamless interfacing with the existing healthcare systems under the criteria of clinical adaptability.

The following is the list of current drivers to translation and implementation: enabling implementation with a common data dictionary and shared language specification; implementation, statistical and clinical safety validation; clinical utility validation; verification, validation, technology assessment; distributed access (federation, cloud computing); simplification and usability; clinician and patient education; sharing best practice; and measuring clinical impact.

The mobile health app categorization and best practices report clusters mHealth applications into the following four categories [10]:

1. General healthcare and fitness

1.1. Fitness \& nutrition

1.2. Health tracking tools

1.3. Managing medical conditions

1.4. Medical compliance

1.5. Wellness (traditional and corporate)

2. Medical Information

2.1. Reference

2.2. Diagnostic Tools

2.3. Continuing Medical Education (CME)

2.4. Alerts and Awareness

3. Remote Monitoring, Collaboration and Consultation

3.1. Remote monitoring (safety)

3.2. Remote Consultation

3.3. Remote Collaboration

4. Healthcare Management

4.1. Logistical \& payment support

4.2. Patient health records

By analysing this list of categories, it is possible to identify a shift towards connected services and interoperable systems. Based on this one could argue that the era of discrete solutions is already behind.

In accordance to the tracking for health report developed in result of the survey by the Pew Research Center's Internet \& American Life Project [11] tracking of health data gradually is getting to be a norm for the U.S. citizens. For example:

$60 \%$ of adults say they track their weight, diet, or exercise routine.

$33 \%$ of adults track health indicators or symptoms, like blood pressure, blood sugar, headaches, or sleep patterns.

$12 \%$ of adults track health indicators or symptoms for a loved one.

Taking into account an absence of specialized healthand wellness-supporting applications a decade ago, this statistics proves a growing demand for serious consumer's applications that operate with biomedical data.

\section{New Basic mHealth Use Cases with Special Focus ON THE DEVELOPING COUNTRIES}

As a starting point for thinking on how to form the new user groups, it is useful to first build good understanding of the typical today's users and analyze what functions and services they are using most actively. Such analysis shall enable us to derive the existing correlations in the age 
categories, social status and so on, and see what are the uncovered needs and user groups.

It is important to find out certain functionality that can be provided with help of mobile device as an inexpensive massmarket solution that addresses critical needs of people. It would be especially beneficial to target first solution to people with low income, as this could be a strong argument for these people to start using mobile devices and this way we can develop a new market niche. The main focus of this section is to initiate discussion around the subject, propose basic principles of the corresponding solutions and study what new functionality they require.

Let's discuss mobile services for developing countries, as nowadays the regions with lowest mobile device penetration are pure countries with undeveloped infrastructure and low level of education. In this group of countries we find many African and Asian countries, including large areas in India and China, some regions of Latin America and even some regions of the former Soviet Union [12]-[16]. One can notice that at the same time these regions are strongly suffering from a number of diseases that can be easily cured if local people would have access to the proper knowledge presented in the easily understandable form. Modern smartphones combine capabilities of telephone, personal computer, Internet access, advanced graphical and audio input and output and so on. From the technical point of view this gives to them the best positioning to take a role of the personal medical assistant. In fact this idea is not new and can be found in a number of earlier publications [17]-[19]. However, we still do not see widespread mobile services that address real problems and targeting developing markets.

The first service idea discussed in this study is a medical consultant (and encyclopedia) based on the image-specified user interface (UI). Already now one can find PC-based medical consultant solutions for professionals [20], [21] Some of the existing solutions have advanced UIs, e.g. with voice UI [22]. However, the UIs of such applications usually tend to reflect high complexity of the underlying subject are, which makes it overcomplicated and often not suitable even for the well educated physicians, and of course it is too complicated for people who hardly can read. Our idea is to take database of one of the existing medical consultant solutions (not only software systems, but also web services are considered), select simplest relevant cases, which have not complicated decision making trees. The selected cases should be encoded by a tree of simple choices represented by images. The UI of proposed solution combines image-based data representation on the screen with touch screen choice selection and audio feedback mechanism. Most of data provided to the user in image format, in some cases it is combined with audio in the native language of the region. The application should also contain embedded configurable phonebook of the local medical centers, where people will be able to get assistance in the native language. It also very important to allow using camera of the mobile device for data input. For example by making photo of the skin surface damaged by a disease or injured some other way. The service should not only allow making pictures, but should interactively ask the user to define on the body map the exact place where this picture was taken. This approach will significantly simplify distant diagnosis, which is a big problem nowadays.

Another application area related to the fact that often people do not know how to render first aid to the person in an emergency situation. The mobile device is constantly with the person and can provide required assistance in rendering the first aid. By starting special application, the user will be able to first by pictures select what type of emergency situation has occurred (e.g. fire, attack by wild animal, etc) then using local emergency database the mobile phone tries to find who can help and generally what to do. After calling help (when it is possible), the mobile device will use own database to give voice instructions of what to do before help arrives. We know that it is important that the person must be focused on the first-aid treatment, but in parallel the device should start search for professionals who could remotely help, e.g. sometimes it might save patient's life if some doctor from other town can provide at least remote general instruction on how to behave. It is well know that in emergency situation a lot of damage comes from wrong behavior of the person who provides first help, which can be a result of lack of knowledge, shock and other reasons.

Another interesting mobile healthcare solution for the developing countries is based on idea of anti-bacterial mechanical protection. One can notice that the main growing markets such as China, India, Indonesia, Brazil and Africa [23] are located in the regions with high bacterial risk. Medical statistic for these counties is showing that the thread of getting and spreading new viruses is increasing all the time. A large share of diseases is spread due to lack of hygiene culture, e.g. via dirty hands. Also in most cases local people don't care of keeping mobile device clean. In the worst case it could turn mobile devices into a carrier for bacteria, which at the event of phone calls get direct access to the user's face and the ear area, which is one of the most sensitive regions of the human body. The local people might notice that mobile device owners more often get skin disease, which could result in wrongly motivated fear of the mobile devices. The proposed solution is to introduce changeable "soap" cover for the mobile devices. The cover should be painted by some antiseptic material that is cheap can be active over reasonably long period of use. An example technology could be SilveGard [24], but its cost still has to be reduced. By introducing this solution we not only fight against the fear of mobile device, but also help to fight against spreading of new viruses thanks to continues contact of the user's hands with the antiseptic material. We 
are planning to continue search for a cheap technology that can provide durable antiseptic painting of the device.

According to the preliminary study, delivering to the developing countries markets a device with the above described services and anti-bacterial mechanical protection will be very much appreciated and could take a large niche of mobile phone sales, a assuming that it will be possible to keep this solution within $10 \%$ cost margin from other basic mobile devices on the market.

\section{Advanced Use Cases: Healthcare Smart Systems}

Implementation of advanced use cases requires use of sophisticated systems for medical data collection and processing. Even a high-level analysis of this topic allows identifying two groups of smart mHealth systems:

1) Personal mobile smart mHealth systems for monitoring user's body parameters in 24/7 mode;

2) Smart mHealth systems that utilize certain patterns of user behavior to make deeper analysis of his/her health condition.

The most natural use case for personal mobile healthcare smart system is a system that performs 24/7 recording of personal health data, making continues analysis of collected data and delivering early diagnostics and other mHealth services to the users, physicians and other relevant parties. Majority of such systems consists of a number of inexpensive medical sensor devices connected (wirelessly) to a personal data processing hub (e.g., smartphone). The system use advanced algorithms for preprocessing of collected medical data, detection of correlations and fuzzy dependencies between the monitored parameters, and building the overall picture using contextual information, such as atmospheric pressure, info on magnetic storms, irritants in the air, geo-location and so on. The whole system could work completely autonomously by providing user with recommendations on healthy behavior, automated treatment in emergency cases (e.g., calling for help) and generating personalized medical record of the user. The collected data has key importance for accurate diagnostics and provide doctors with most accurate picture for the individual case and patients with relevant recommendations when they are most relevant. Our team is already actively working on development of mobile diagnostics solution and this work will continue.

Nowadays one can find also a number of solutions that belong to the second group of smart mHealth systems. For example, very popular smart system of this group is a health monitoring system integrated to a toilet seat [25]. But for this paper we would like to particularly see how to efficiently deploy and use smart mHealth systems on board of commercial airlines.

There are well-known flights related healthcare problems. For example, long sitting with little movement could lead to wicking of legs. The blood stasis in the legs veins could result in formation of venous thrombi. In the worst case it could event lead to thrombophlebitis, which is extremely dangerous and might result in death. Another problem is how to increase humidity to the normal level and ensure good ventilation, which would keep level of CO2 on appropriate level, so that passengers will feel less tired. We proposing to use personal onboard smart mHealth systems integrated to passenger's chair for addressing the problems.

Moreover, one could notice that time of flight in aircraft provides good opportunity for general health monitoring. Passenger spends most of time in the chair with minimal movements, which could be used for reasonably long-term monitoring of various health parameters, e.g., heart activity, blood circulation, etc. What makes results even better is that passenger experiences physical and psychological stress, related to events like takeoff, change cabin pressure with altitude, events of turbulence, etc. Early diagnostics of some health problems need monitoring changes of the key body parameters under stress, i.e., see reaction to particular events (which could be accurately detected from contextual records of meters that are already available onboard) and comparing the results to the values in normal conditions. Most of people don't want to visit doctors to take such tests, especially if nothing hurts yet. Conditions in aircraft allow making such monitoring in the most comfortable way for the user. After end of the flight passage shall be able to use personal mobile device to securely collect results of monitoring, add them to the personal medical record and later make use of them for automated health monitoring algorithms and/or make the available for the doctor.

Development of such mHealth medical solution is a huge opportunity and we are planning to launch such project as soon as will make an agreement with an airline partner.

\section{DISCUSSION AND CONCLUSIONS}

Implementation of the mHealth technologies is very beneficial for the clinical medicine. A report by the Veterans Health Administration in the US titled "Care Coordination/Home Telehealth: The Systematic Implementation of Health Informatics, Home Telehealth, and Disease Management to Support the Care of Veteran Patients with Chronic Conditions” reports 25\% reduction in the average number of bed/days for admissions for chronic conditions, through the use of a Home Health Monitoring system and a reduction of hospital admissions by $19.74 \%$ for chronically ill patients monitored from home through a home health monitoring system.

Nearly 40 million yearly hospital admissions are reported collectively in six countries of the EU (Czech Republic, France, the Netherlands, Sweden, Spain and the United Kingdom). It is generally estimated that between $75 \%$ and $85 \%$ of hospital admissions are for chronically ill patients. Assuming that an average of $80 \%$ of hospital 
admissions could be for chronic conditions in all member states, the yearly number of admissions for chronically ill patients could be as high as 30 million collectively in all six countries [26]. Unfortunately it is not possible to find similar figures for fast growing economies, but it is expected that numbers for Russia will be almost the same, while for other BRIC countries are only a bit smaller.

This work is focused on presenting and discussing use cases and the corresponding mass-market healthcare and medical solutions for mobile devices for the selected groups of users. The paper was not targeted to address complex technical, medical and sensors-related issues, instead we focused on identifying user demands and find simple solutions, which can be delivered with reasonable amount of efforts, but will allow significantly changing users' life.

In conclusion we want to stress that the discussed above solutions and corresponding use case scenarios shall not be seen as a replacement of professional medical examination with use of professional medical equipment. But new mHealth services will bring strongly demanded medical services closer to the mass user and they can be seen as an efficient consultancy solution that helps to detect health problem at the early stage and provides reasonably efficient solutions for many annoying problems. As a result we are targeted to provide people with new valuable mass services and so significantly expand base of the mobile devices active users, as the medical and healthcare services are needed for all categories of users independently of their age, gender, location and social status. Health of the nation is the main priority for in the absolute majority of the countries, so in-depth analysis of mass-healthcare use cases and solutions shall be seen as long-term priority. Already now we can see that governments provides a lot of grants and public programs to support development of the corresponding solutions and in the coming years we will see major breakthrough in this area. Also it is important to mention that the proposed solutions directly benefit insurance companies, so potentially some support might also come from their side. The additional support might come from social responsibility programs executed by large industrial companies. Moreover, many companies might be interested to financially support use of this kind of applications by their employees, as it will help to reduce losses due to illness work breaks of the employees.

When people talk about the future of health care, they often have in mind a model of Kaiser Permanente (USA) center. Mr. Halvorson, chairman and chief of Kaiser Permanente, is convinced that Kaiser's improvements in the quality of care save money. But he also says that the way to get costs lower is to move care farther and farther from the hospital setting - and even out of doctors' offices. Kaiser is experimenting with ways to provide care at home or over the Internet, without the need for a physical office visit at all. He also argues that lower costs are going to be about finding ways to get people to take more responsibility for their health - for losing weight, for example, or bringing their blood pressure down [27].

Based on the performed study we believe that nowadays one of the biggest enablers for devices, applications and services to appeal to the huge new groups of users is by creating simple, cheap and mass medical and healthcare solutions on top of the discussed and other new use cases. We are planning to use finding of this study for development of mHealth solutions that fulfill the main need of all people - to be healthy and provide relatives with the proper healthcare protection.

\section{REFERENCES}

[1] "Sustainable Health Systems Visions, Strategies, Critical Uncertainties and Scenarios Report from the World Economic Forum," 2013.

[2] Maryn McKenna, "The New Age of Medical Monitoring: Mobile phones and tiny sensors are making it easier to quickly flag health trends", Scientific American, pp. 33-34, March 2013.

[3] "FCC to Act On Key mHealth Task Force Recommendations to Spur Adoption of Wireless Health Technology", URI http://www.fcc.gov/document/fact-sheet-mHealth-task-forcerecommendations.

[4] "FCC mHealth Task Force Findings and Recommendations: Improving Care Delivery Through Enhanced Communications Among Providers, Patients and Payers”, Pre-publication draft.URI: http://www2.itif.org/2012-mHealth-taskforcerecommendations.pdf. September 2012.

[5] "Mobile Health Trends and Figures 2013-2017", Research2Guidance, July 2013, URI: http://www.researchandmarkets.com/reports/2603873/mobile_healt h_trends_and_figures_20132017.

[6] “The Global Use of Medicines: Outlook through 2017”, 2013.

[7] D. Levi, “Analysis and trends in mHealth,” 2013.

[8] V. Díaz, M. Viceconti, V. Stroetmann, and D. Kalra, "Roadmap for the Digital Patient," 2013.

[9] V. Kramar, M. Korhonen, and Y. Sergeev, "Particularities of Visualisation of Medical and Wellness Data through a Digital Patient Avatar”, in FRUCT 14 Conference, 2013.

[10] Research2Guidance, "Mobile Health App Categorization \& Best Practices,” 2013.

[11] F. Susannah and D. Maeve, “Tracking for Health,” 2013.

[12] E. Tomas, L.A. Facchini and M.D. Maia, "Health information technology in primary health care in developing countries: a literature review,” Bulletin of the World Health Organization, 2004.

[13] P.W. Setel, L. Saker, N.C. Unwin, Y. Hemed, D.R. Whiting and H. Kitange, "Is it time to reassess the categorization of disease burdens in low-income countries?”, American Journal of Public Health, Vol. 94, No. 3, pp 384-388, March 2004.

[14] A. Geissbuhler, O. Ly, C. Lovis and J. L'Haire, “Telemedicine in Western Africa: lessons learned from a pilot project in Mali, perspectives and recommendations," in proceedings of the AMIA Fall Symposium 2003.

[15] "The difficult case: Healthcare in developing countries," publication at http://medicinform.net/medmir/raz.htm, 2010. (in Russian)

[16] N.V. Urgel and M.Y. Xubieva, "On improving the quality and accessibility of medical care in the country side areas,” publication at http://www.remedium.ru/section/state/detail.php?ID=20342. 
[17] O. Medvedev, A. Kobelev, S. Schookin, M. Jatskovsky, G. Markarian and I. Sergeev, "Smartphone-based Approach for Monitoring Vital Physiological Parameters in Humans”, IFMBE Proceedings, Springer. Vol. 14, Number 6, pp. 4020-4022, 2007.

[18] O. Medvedev, A. Marshall and A. Antonov, "User-Friendly Interface for the Smartphone-based Self Management of Pulmonary Rehabilitation”, in proceedings of 2008 International Conference on BioMedical Engineering and Informatics, pp. 673-676, 2008.

[19] C. Thwaites, "Mobilising medical support," publication at http://ictupdate.cta.int/en/Regulars/Perspectives/Mobilisingmedical-support, ICT Update, Issue 48, April 2009.

[20] Dragon Naturally Speaking Software Suite Transcription Products, publication at http://www.dragon-medicaltranscription.com/transcription_PRODUCTS.html, 2010.

[21] Medical Software Associate, “Consultant EMR software”, publication at http://www.medical-software.org/consultant-emrsoftware.html, 2010.

[22] Sofotex, “American Medical Encyclopedia,” publication at http://www.sofotex.com/American-Medical-Encyclopediadownload_L4548.html, 2006.

[23] R. Loewenson and D. McCoy, “Access to antiretroviral treatment in Africa,” British Medical Journal, Vol. 328(7434), pp. 241-242. 2004.

[24] Medical Device Infection Control, "SilvaGard Surface Engineered Antimicrobial Treatment," AcryMed, publication at http://www.acrymed.com/medical.html, 2010.

[25] "Smart Toilets, a royal flush for Home Healthcare?", Modern Health Talk, URI: http://www.mHealthtalk.com/2011/04/smart-toilets-aroyal-flush-for-home-healthcare/. 2011.

[26] “eHealth for a Healthier Europe!”, Gartner report. URI: http://www.government.se/sb/d/574/a/129815, 2009

[27] "The Face of Future Health Care", The New York Times, URI: http://www.nytimes.com/2013/03/21/business/kaiser-permanente-isseen-as-face-of-future-health-care.html?pagewanted=all\&_r=1\&. March 21, 2013. 